\title{
SOCIOLOGIA DA DEFICIÊNCIA: VOZES POR SIGNIFICADOS E PRÁTICAS (MAIS) INCLUSIVAS
}

\author{
Franco Ezequiel HARLOS ${ }^{1}$ \\ Fátima Elisabeth DENARI ${ }^{2}$
}

RESUMO: Na presente pesquisa objetiva-se sistematizar histórias e teorias associadas com a Sociologia da Deficiência, e identificar, nestes elementos sistematizados, vozes para repensar o significado da deficiência e práticas da Educação Especial. Para tanto, percorreu-se na pesquisa bibliográfica e documental 48 obras compiladas, primordialmente, da revista Disability and Society, da Asociación Española de Sociología de la Discapacidad e da base de dados do Centre of Disability Studies da Universidade de Leeds. As obras compiladas foram analisadas por meio de análise categorial. A primeira das categorias identificadas centra-se nas principais interpretações adotadas pelas Ciências da Saúde (paradigma médico) para pensar a deficiência; a segunda, em histórias associadas com a constituição da Sociologia da Deficiência - nos movimentos sociais que, em resposta às interpretações da deficiência adotadas pelas Ciências da Saúde, (re)significaram a deficiência e fundaram os Estudos da Deficiência (Disability Studies); e, a terceira, nas múltiplas faces teóricas da Sociologia da Deficiência e nos novos modelos de compreensão da deficiência. A sistematização do conteúdo desvelou um paradigma sociológico de interpretação da deficiência: este paradigma apresenta vozes por significados e práticas mais inclusivas.

PALAVRAS-CHAVE: Sociologia da deficiência. Estudos da deficiência. Educação especial. Pessoas em situação de deficiência.

\section{Introdução}

A Educação Especial conta com poucos instrumentos teóricos, discursivos e conceituais para a análise dos aspectos sociais da deficiência: na Educação Especial faltam ferramentas analíticas para se entender como certas configurações educacionais e sociais produzem a categoria deficiente e reproduzem as condições de pobreza e segregação, a que muitas pessoas em situação de deficiência estão submetidas. Aliás,

\footnotetext{
1 Doutorando em Educação Especial. UFSCar - Universidade Federal de São Carlos. Centro de Educação e Ciências Humanas - Pós-Graduação em Educação Especial. São Carlos - SP - Brasil. 13565905. Integrante da Asociación Española de Sociología de la Discapacidad (ASESDIS). Professor. IFPR Instituto Federal de Educação, Ciência e Tecnologia. Foz do Iguaçu - PR - Brasil. 85860-000 franco.harlos@ifpr.edu.br.

${ }^{2}$ Professor Associado do Departamento de Psicologia. UFSCar - Universidade Federal de São Carlos. Centro de Educação e Ciências Humanas. São Carlos - SP - Brasil. 13565-905 - fadenari@ terra.com.br.
} 
Educação Especial e deficiência foram historicamente pensadas a partir do referencial das Ciências da Saúde.

Paradoxalmente, as propostas de Inclusão Escolar de pessoas em situação de deficiência suscitam ampla compreensão dos aspectos sociais da deficiência. Neste meandro, no campo da Educação Especial, observa-se a demanda por vozes ${ }^{3}$, por ferramentas discursivas que tenham sua gênese em fontes distintas daquelas que predominantemente são adotadas para pensar significados e práticas associadas com as pessoas em situação de deficiência.

Neste contexto, no presente estudo se aposta que na Sociologia da Deficiência existem múltiplas vozes que apresentam léxicos e categorias discursivas, para a interpretação dos elementos da deficiência para os quais, na Educação Especial, existem poucas ferramentas analíticas.

A partir desta aposta, demarcam-se os objetivos da presente pesquisa, a saber: sistematizar histórias e teorias associadas com a Sociologia da Deficiência, e identificar, nestes elementos sistematizados, vozes para repensar o significado da deficiência e as práticas da Educação Especial voltadas às pessoas em situação de deficiência. "Novas" vozes por significados e práticas (mais) inclusivas.

Com estes objetivos, com base em pesquisa teórica pautada em pesquisa bibliográfica e documental, foram sistematizados aspectos da história dos movimentos sociais denominados Union of the Physically Impaired Against Segregation e Independent Living Movement, e aspectos das teorias sociológicas indicadas na revista Disability and Society e na Asociación Española de Sociología de la Discapacidad, pois são estas histórias e teorias que estão especialmente intrínsecas à constituição da Sociologia da Deficiência e fundam uma polifonia de novas possibilidades analíticas quanto à deficiência. Neste ínterim, nas linhas que seguem, expõe-se o conteúdo, os resultados e percurso da presente pesquisa.

\section{Método}

Para a compilação de materiais para a constituição do corpus bibliográfico da presente pesquisa, inicialmente, utilizou-se duas fontes, a saber: a revista Disability and

\footnotetext{
3 Na presente pesquisa utiliza-se a expressão vozes para a alusão de teorias, conceitos, princípios ideológicos, axiomas, palavras, histórias ou outros elementos discursivos que possam instrumentalizar e fundamentar revisões de significados associados com a deficiência e/ou com a Educação Especial.
} 
Society $^{4}$ - D\&S e a base de dados da Asociación Española de Sociología de la Discapacidad - ASESDIS 5 .

Para a seleção de publicações nestas fontes adotou-se os seguintes critérios:

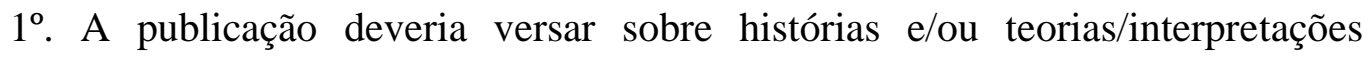
relacionadas com a Sociologia da Deficiência.

$2^{\circ}$. Cada publicação compilada, em relação às obras já selecionadas, deveria apresentar um novo aspecto das histórias e/ou teorias/interpretações relacionadas com as análises sociológicas da deficiência;

$3^{\circ}$. Não preenchendo nenhum dos critérios anteriores, a publicação só poderia ser selecionada caso fosse frequentemente citada (nas obras já selecionadas) como "marco" histórico do processo de delimitação de uma perspectiva sociológica de interpretação da deficiência e/ou como obra que estabelecia uma nova leitura sociológica em relação à deficiência.

No processo de seleção de artigos da revista D\&S duas coletâneas de publicações da revista D\&S, organizadas por Barton $(1998,2008)$ foram determinantes. Segundo Barton (1998, 2008), editor da revista em questão, estas coletâneas buscam apresentar um panorama dos artigos que tiveram maior impacto nos estudos sociológicos da deficiência, publicados na revista em questão, bem como reúnem artigos que marcam, respectivamente, a primeira e a segunda década da revista.

A partir das considerações de Barton (1998, 2008), da leitura dos 33 artigos que compõem estas coletâneas, de acordo com os critérios supracitados, selecionou-se 14 destes artigos e interpretou-se que os mesmos seriam um ponto de partida mais adequado à compilação de materiais para a presente pesquisa, do que o uso de descritores para buscas na base da revista $D \& S$.

Quanto às publicações da ASESDIS, efetuou-se leitura de todos os artigos que estavam disponibilizados em sua homepage na época da coleta (entre junho e setembro de 2011) e deles selecionou-se 11 artigos que correspondiam aos critérios supracitados.

Da leitura do material compilado da revista D\&S e a partir das publicações da ASESDIS, selecionou-se 23 publicações apontadas como partes fundamentais da história da Sociologia da Deficiência. Coletou-se 10 destas últimas obras mencionadas

${ }^{4}$ Disponível em:<http://www.tandfonline.com/loi/cdso20\#.UtluaxC5fIU〉. Acesso em: 22 abr. 2015.

${ }^{5}$ Disponível em: $<$ http://www.um.es/discatif/PROYECTO_DISCATIF/Discatextos_1.htm>. Acesso em: 22 abr. 2015. 
por meio do banco de dados do Centre of Disability Studies ${ }^{6}$ - CDS, da Universidade de Leeds, e 13 de fontes diversas.

Assim efetuou-se a compilação de um total de 48 obras.

O Quadro 1 retrata a totalidade de obras compiladas, por fonte, autor e ano de publicação.

Quadro 1 - Corpus bibliográfico estruturante

\begin{tabular}{|c|l|c|}
\hline Fontes & \multicolumn{1}{|c|}{ Obras } & Total \\
\hline D\&S & $\begin{array}{l}\text { Abberley (1998); Barnes(1998); Barton (1998); Oliver (1998); Peters (1998); } \\
\text { Thomas (2004); Abberley (2008); Barnes(2008); Hanna e Rogovsky (2008); } \\
\begin{array}{l}\text { Hughes e Paterson (2008); Morris (2008); Oliver (2008); Peters(2008); } \\
\text { Shakespeare(1997) e Shakespeare(2008). }\end{array}\end{array}$ & 14 \\
\hline ASESDIS & $\begin{array}{l}\text { ASESDIS (2002); López (2006); Palacios e Romañach (2006); Ferreira (2007, } \\
\text { 2008, 2010); Ferrante e Ferreira (2008, 2010); Palacios e Romañach (2008); } \\
\text { Díaz(2009); Rodrigues e Ferreira (2010); }\end{array}$ & 11 \\
\hline CDS & $\begin{array}{l}\text { Hunt (1966, 1981); UPIAS (1976); Finkelstein (1980); Barnes (1991, 2007); } \\
\text { Oliver(1994, 1996); Shakespeare e Watson (2002); Pfeiffer (2002); }\end{array}$ & 10 \\
\hline OUTROS & $\begin{array}{l}\text { Goffman (1974, 1975); Parsons (2005); Foucault (2001, 2008) Garcia (2003); } \\
\text { Barnes (2003, 2009); Osamu(2005); Barton (2009); Finkelstein (2007); } \\
\text { Brogna(2009); Taylor et al. (2009); DPI(2010) }\end{array}$ & 13 \\
\hline
\end{tabular}

Fonte: Elaboração própria.

A análise de conteúdo foi empregada para análise destas obras compiladas, sendo compreendida como um conjunto de técnicas de análises das comunicações marcado por uma grande disparidade de formas e adaptável a todo o vasto campo das comunicações (BARDIN, 2008). A técnica de análise de conteúdo utilizada foi a análise categorial e as categorias identificadas a partir destas técnicas foram as seguintes: 1. Paradigma Médico de interpretação da deficiência; 2. Histórias associadas com a Sociologia da Deficiência; e, 3. Teorias da Sociologia da Deficiência.

Por fim, para tratamento e interpretação dos resultados, sistematizou-se e comparou-se o conteúdo das diferentes unidades destacando os elementos que permitem caracterizar a Sociologia da Deficiência e repensar significados e práticas associados com a Educação Especial e com as pessoas em situação de deficiência.

${ }^{6}$ Disponível em:<http://disability-studies.leeds.ac.uk/publications/>. Acesso em: 22 abr. 2015. 


\section{Resultados e discussões}

\section{paradigma médico de interpretação da deficiência}

Nesta categoria agruparam-se considerações sobre interpretações das Ciências da Saúde em relação à deficiência - que incitaram críticas e reinterpretações da deficiência pautadas na Sociologia e assim contribuíram para o desenvolvimento da Sociologia da Deficiência. Versa-se também sobre um Modelo Individual e um Modelo Biopsicossocial de compreensão da deficiência, bem como, aborda-se uma descrição breve do conteúdo da Classificação Internacional de Funcionalidade, Deficiência e Saúde - CIDID.

A partir de Oliver (1996), WHO (2001), López (2006), Barnes (2009), Díaz (2009), Ferreira (2007, 2008, 2010), Ferrante e Ferreira (2007, 2008), identificou-se dois modelos de deficiência associados a Ciências da Saúde: o Modelo Individual e o Modelo Biopsicossocial.

$\mathrm{Na}$ lógica de raciocínio que sustenta o modelo individual, fundamentada especialmente na CIDID, a deficiência é interpretada como um problema individual que se origina de uma doença ou distúrbio, que se manifesta na perda ou anormalidade de uma estrutura ou função psicológica ou corporal e, por si só, provoca desvantagens sociais. A origem das desvantagens sociais estaria então nas próprias pessoas em situação de deficiência, nas condições corporais ou intelectuais que as tornariam inaptas para as atividades sociais.

Para Oliver (1996), no Modelo Individual de compreensão da deficiência, o problema implícito é a própria pessoa com deficiência, condição tal que se encontra em um esquema discursivo justificado pelas seguintes expressões: caso médico, sintomas, cura, amenização, profissionais da saúde, cuidados, não anda, não fala, não vê, não decide, instituições especiais, assistência social, emprego protegido, escolas especiais, transportes especiais, hospitais, assistentes sociais, entre outras. Para Oliver (1996), López (2006), Barnes (2009), Díaz (2009), Ferreira (2007, 2008, 2010), Ferrante e Ferreira (2007, 2008), a lógica que orienta o Modelo Individual não deve ser aceita em uma perspectiva sociológica ou inclusiva, justamente porque a mesma desconsidera os aspectos sociais produtores da deficiência.

Por sua vez, no modelo biopsicossocial de deficiência apresentado no documento International Classification of Functioning, Disability and Health - ICF a 
"deficiência é o desfecho ou o resultado de uma complexa relação entre o estado de saúde do indivíduo e fatores pessoais e, fatores externos que representam as circunstâncias em que o indivíduo vive" (WHO, 2001, p.17). Ou seja, na ICF, deficiência denota os aspectos negativos da interação entre os seguintes fatores: estado de saúde, fatores pessoais e externos.

Para Barnes (2009), que prefere denominar o modelo em questão como Modelo Inter-relacional, o problema do modelo é justamente de que nele a deficiência segue sendo, mais do que um assunto político, um assunto da saúde. Esta interpretação de Barnes (2009) é semelhante aos dos demais autores citados neste tópico e foi constantemente criticada na história de construção desta face sociológica dos Estudos da Deficiência: as histórias associadas com a Sociologia da Deficiência são sintetizadas na unidade seguinte.

\section{Histórias associadas com a Sociologia da deficiência}

Nesta categoria agruparam-se os aspectos históricos dos movimentos sociais das pessoas em situação de deficiência que fundam bases para que conceitos de deficiência e Educação Especial sejam repensados. No final dos anos sessenta, diversos países ocidentais viviam, simultaneamente, novos e fortes movimentos sociais. Estes movimentos reivindicavam os direitos de grupos específicos como negros, mulheres e índios e influenciavam-se mutuamente no sentido de que os avanços e conquistas de um deles impulsionavam a luta dos outros por conquistas similares ou por novas conquistas. Nesse período, a politização das pessoas em situação de deficiência e dos familiares das mesmas também se fazia sentir.

Em países da América Latina, da América do Norte, da África e da Europa, formavam-se movimentos sociais voltados para a reivindicação dos direitos das pessoas em situação de deficiência, os denominados Disabylity Rigths Movement. Na Inglaterra, nascia o movimento denominado Union of The Physically Impaired Against Segregation - UPIAS e nos Estados Unidos da América, Independent Liveng Movement - ILM, ambos organizados por pessoas em situação de deficiência (SHAKESPEARE, 2008).

Inicialmente o problema identificado por UPIAS e ILM foi a marginalização opressiva das pessoas em situação de deficiência e as origens destes problemas foram apontadas nas ideias e práticas dominantes, na estrutura social hegemônica, no Modelo 
Individual de deficiência e nas barreiras sociais, físicas, econômicas e atitudinais em relação a este coletivo.

Para combater estes problemas, os movimentos sociais em questão atacaram em três frentes: epistemológica, legislativa e organizativa. Em termos epistemológicos as respostas aos problemas sociais foram a criação de novos significados e modelos relacionados com a compreensão da deficiência e com os fatores que incapacitam pessoas que apresentam diferenças corporais ou intelectuais que convencionalmente são caracterizadas como deficiência (SHAKESPEARE, 2008).

Da UPIAS nascia uma interpretação sociológica da deficiência, denominada Modelo Social de compreensão da deficiência. Do ILM e do próprio Modelo Social, nascia outro modelo baseado na defesa dos direitos civis e humanos das pessoas em situação de deficiência, um modelo denominado Modelo Baseado em Direitos.

Em termos legislativos, lutou-se pela promulgação de leis que impedissem ou combatessem os problemas enfrentados pelas pessoas em situação de deficiência. Leis estas marcadas pela tentativa de promover a inclusão das pessoas em situação de deficiência na sociedade, nas escolas, pela tentativa de operacionalizar vagas específicas para as pessoas em situação de deficiência (por exemplo, as leis de cotas) e de proibir atitudes discriminatórias e preconceituosas em relação ao coletivo em questão.

Por um lado, o avanço da organização destes dois movimentos (UPIAS e ILM) incitou uma quantidade crescente de pessoas em situação de deficiência a adotar uma identidade política compartilhada e, a partir desta identidade, muitas destas pessoas já não pediam mudanças, as exigiam e se dispunham a lutar por elas (SHAKESPEARE, 2008). Por outro lado, “a auto-organização e ação direta desafiavam os estereótipos reinantes de impotência e coisificação" (SHAKESPEARE, 2008, p.84) relacionados com as pessoas em situação de deficiência. Ao converterem os problemas que os assolavam em assuntos públicos, as pessoas em situação de deficiência vinculadas a estes movimentos afirmavam a validade e a importância de sua própria identidade e possibilitavam a (re)significação desta em diversos contextos (SHAKESPEARE, 2008).

Desde que estes dois movimentos sociais se "entrelaçaram" política e ideologicamente, especialmente com a fundação da Disabled Peoples' International$D P I$, novos significados se consolidam globalmente para aquilo que entende-se como deficiência e novos campos do saber se estruturam para aprimorar estas interpretações. 
Assim, a partir destes movimentos, teorias sociológicas sobre a deficiência foram constituídos e/ou re-interpretadas, formando uma sociologia específica denominada Sociologia da Deficiência: esta sociologia específica que tem amplo potencial para provocar revisões em práticas educativas e sociais relacionadas com as pessoas em situação de deficiência.

\section{Teorias da Sociologia da deficiência}

Nesta categoria foram agrupadas informações sobre algumas obras que permitem vislumbrar as distintas perspectivas teóricas e analíticas adotadas nos estudos relacionados com a Sociologia da Deficiência, bem como se descreve sinteticamente clássicos da Sociologia que tem especial impacto nos estudos sociológicos da deficiência.

Destaca-se que as interpretações sociológicas da deficiência dialogam com estudos sobre doenças, estigmas, a anormalidade, a exploração, o racismo e as questões de gênero e, por vezes, apresentam interpretações da deficiência com cisões similares às demarcadas entre sexo (características anatômicas e hormonais de uma pessoa) e gênero (expressão culturalmente apropriada de masculinalidade e feminilidade), raça (marcas físicas, corporais) e etnicidade (marcas culturais).

Vislumbra-se então que, no que se refere às interpretações sociológicas que precederam a Sociologia da Deficiência e foram importantes para a constituição desta, as interpretações de Parsons (2005), Goffman (1974, 1975) e Foucault (2001, 2008) tiveram um papel especial e estão dentre as primeiras vozes que, direta ou indiretamente, subsidiaram análises sociológicas da deficiência.

As interpretações de Parsons (2005) apresentaram os primeiros indícios das relações de poder que permeiam as interações entre médicos e doentes e/ou deficientes; e, constituem reflexões sobre como os papéis sociais atribuídos para os doentes tem um tom de submissão, de necessária aceitação dos ditames da medicina. Estas reflexões parsonianas podem instrumentalizar reflexões sobre, por exemplo, os papéis sociais de educador especializado e "aluno especial" com deficiência e/ou sobre as relações de poder que se manifestam na interação entre pessoas com estes distintos papéis sociais.

Com Goffman $(1974,1975)$, dentre outros elementos, foram desvelados o papel dos estigmas, das marcas que sendo socialmente registradas em alguns corpos, alteram as relações sociais que se estabelecem com os estigmatizados. E, também foram discutidas as funções das instituições totais, que tantas vezes se fazem vivas em 
ambientes organizados para a educação e/ou reabilitação de pessoas em situação de deficiência. Assim, as análises de Goffman $(1974,1975)$ e os conceitos que ele constituiu são ferramentas analíticas importantes para que sejam (re) pensadas, tanto as relações entre pessoas em situação de deficiência e as demais pessoas, quanto o próprio significado da deficiência.

Por sua vez, as interpretações de Foucault (2001, 2008), ao produzirem uma reconstrução genealógica do conceito de "anormal", uma genealogia da anomalia e do indivíduo anormal, dos dispositivos que servem à definição do mesmo e das tecnologias de poder que lhe correspondem, descortinaram processos que estão na gênese da anormalidade e da própria constituição da categoria deficiente; bem como, permitiram a fundação de novos olhares sobre as relações de poder-saber que incidem sobre pessoas em situação de deficiência no cotidiano das práticas escolares e médicas voltadas a este coletivo. Por essas características as análises foucaultianas são um referencial indispensável para (re)pensar-se práticas e discursos que levam a deficiência para o território da anormalidade e/ou fundam mecanismos disciplinares com forte incidência negativa para o coletivo em questão.

Quanto aos pesquisadores vinculados com Estudos da Deficiência e com a UPIAS e o ILM, observa-se que a partir de Hunt (1966, 1981), foram cimentados os pilares basilares para a negação de um discurso trágico sobre deficiência e para a percepção da importância de que as pessoas em situação de deficiência sejam partícipes ativos das pesquisas, políticas e/ou quaisquer outras propostas que sobre elas versem. Estes pilares, até hoje são bases fundamentais dos princípios do ILM, e, portanto, ainda são recursos importantes para confronto às propostas que retiram das pessoas em situação de deficiência o direito de independência e participação social.

Com Finkelstein (1980), Oliver (1990) e outros olhares marxistas, dentre outros aspectos, os estudos em questão desvelaram que as pessoas em situação de deficiência também vivem os ditames do capitalismo e, quando integrantes das classes menos favorecidas, experimentam a condição específica de terem sua pobreza aumentada por só terem o trabalho para vender e não poderem vendê-lo por que desacreditados de suas possibilidades ou porque efetivamente incapazes de trabalharem da mesma maneira que os demais membros da sociedade. Estes referenciais marxistas são significativas vozes para análise das possibilidades da Inclusão Escolar de pessoas em situação de deficiência e os nuances específicos desta inclusão na sociedade capitalista; bem como, 
para entender-se como questões profissionais e econômicas estão atreladas à produção da deficiência na atualidade.

Os estudos de Abberley (1998, 2008) e Barnes (1991, 1998) aproximaram a experiência de opressão por deficiência com as experiências de outros coletivos oprimidos e indicaram especificidades desta experiência e elaboraram novos instrumentos teórico-analíticos de reflexão sociológica sobre deficiência, como o conceito de deficientismo (disablism, conceito análogo aos conceitos de racismo e sexismo, porém relacionado com o preconceito contra pessoas em situação de deficiência) e os estudos sobre a discriminação institucionalizada contra pessoas em situação de deficiência. Além disso, incitaram novas reflexões sobre a cultura da deficiência, como elemento importante na caracterização do coletivo em questão.

Assim, seja apresentando novos conceitos e/ou subsidiando reflexões sobre uma possível cultura da deficiência, Abberley (1998, 2008) e Barnes (1991, 1998), ampliam o leque de possibilidades analíticas da deficiência produzindo instrumentos teóricos e discursivos que, na Educação Especial, podem ser fundamentais para se pensar elementos que vão das práticas de discriminação institucionalizada às manifestações culturais do grupo de pessoas em situação de deficiência.

Com os estudos feministas, como o de Morris (2008) e Hanna e Rogovski (2008), foram desveladas não só as implicações das relações entre gênero, deficiência e outras variáveis de desigualdade (raça, orientação sexual ou idade), como a importância do cuidado, das cuidadoras e da percepção da interdependência entre os seres humanos, na constituição dos direitos das pessoas em situação de deficiência. Na Educação Especial, estas ideias são recursos cruciais para instrumentalizar um (re)pensar das relações entre cuidado e educação e as interdependências entre educador e educando.

Shakespeare e Watson (2002) e Hughes e Paterson (2008) evidenciaram a problemática da distinção entre lesão e deficiência, existente no Modelo Social e em grande parte dos estudos da Sociologia da Deficiência, bem como apontaram a unicidade destes dois fenômenos. Com estas análises, forneceram ferramentas para que a deficiência seja compreendida em sua integralidade: ou seja, para que sejam reduzidas as subdivisões disciplinares que transformam a experiência da deficiência em uma série de elementos administráveis por distintas áreas do conhecimento.

Além disso, tiveram um papel importante na constituição de novos estudos sobre o corpo com lesões, como os estudos de Ferrante e Ferreira (2008, 2010), Ferreira (2008, 2010), Rodriguez e Ferreira (2010), que reforçam a atenção à dimensão 
corpóreo-social da deficiência - dimensão esta que embora a Educação Especial não negligencie, muitas vezes analisa apenas do ponto de vista biofisiológico, desconsiderando a construção social do corpo.

Todo esse processo levou a constituição de novos modelos de compreensão da deficiência como o modelo da diversidade funcional, que posiciona deficiência como uma das manifestações da diversidade humana. Palacios e Romañach $(2006,2008)$, os criadores deste modelo, também pontuam que é imprescindível que se acabem com os paradoxos terminológicos presentes em expressões como eficiência, deficiência, e que sejam criados termos que denotem uma identidade não negativa para o coletivo em questão, tais como: pessoas com diversidade funcional, diversidade funcional auditiva, diversidade funcional intelectual, diversidade funcional intelectual, mulheres e homens com diversidade funcional.

Estes modelos são, por si só, novas vozes que podem instrumentalizar propostas que vão de políticas públicas a práticas educacionais voltadas para o coletivo em questão, fundamentando ações mais adequadas para que os direitos humanos das pessoas em situação de deficiência sejam respeitados.

Neste contexto, novas palavras ou expressões como pessoas com diversidade funcional e deficientismo, são verdadeiros instrumentos constituídos para análise do significado deficiência e/ou das políticas voltadas para as pessoas em situação de deficiência. Aliás, mesmo que estas expressões sejam consideradas inadequadas, o simples debate sobre elas põe em cena inúmeros aspectos da deficiência não vislumbrados no Paradigma Médico, como a relação intrínseca entre deficiência e as outras faces da diversidade humana.

Estes modelos também indicam que a Sociologia da Deficiência se desenvolveu em constante diálogo com as tentativas de reinterpretação da deficiência; e, que esta sociologia específica tem se constituído terreno fértil para a formação de significados para a deficiência. Aliás, em todo o compendio de teorias sistematizadas observamos o tom crítico e ativista das discussões promovidas pelo ILM e pela UPIAS e percebe-se que o corpus teórico associado à Sociologia da Deficiência questiona os significados atribuídos à deficiência.

Concorda-se com Barton (1998) quando destaca que a Sociologia da Deficiência implica em análises de questões de poder e justiça social, da cidadania e dos direitos humanos, bem como implica na formulação de perguntas fundamentais sobre as condições e as relações estruturais que estabelecem e legitimam a criação de barreiras 
sociais que se interpõe entre pessoas em situação de deficiência e a sociedade como um todo.

Desta forma, entende-se que a Sociologia da Deficiência é composta por um arranjo de distintas análises, que renovam e adaptam teorias para formularem explicações sobre aspectos sociais relacionados com as pessoas em situação de deficiência; e, por um conjunto de interpretações da deficiência em diversas perspectivas de análise sociológica, que explicitam que a deficiência não pode ser considerada apenas um problema médico que afeta uma minoria da população, mas deve ser entendida como "uma questão central para integrar o discurso sociológico e análise de outras categorias sociológicas como classe, raça, gênero e sexualidade" (BARNES; OLIVER, 1991, p.2).

Com estes elementos caracterizantes e constituintes, porque não pode ser explicada do mesmo marco interpretativo, entende-se que a Sociologia da Deficiência constitui âmbito de estudo complementar, mas independente de outros campos de saber, como a Educação Especial, a Psicologia e a Medicina; e, entende-se que "o tema mais importante da Sociologia da Deficiência é conceitual" (ABBERLEY, 1998, p.76), pois a mesma estrutura um novo caminho possível para interpretação da deficiência. Denomina-se este caminho como Paradigma Sociológico de Interpretação da Deficiência.

\section{Conclusões}

Seja com as teorias antecedentes ou constituintes da Sociologia da Deficiência ou com a identificação/caracterização dos elementos constituintes deste campo, confirma-se que a sociologia específica em questão apresenta um arcabouço teóricoconceitual-discursivo fundamental para que a deficiência possa ser pensada por novas óticas, como a do Paradigma Sociológico de Interpretação da Deficiência; para que educadores especiais e pesquisadores da Educação Especial ampliem seus instrumentos teóricos e analíticos, no sentido de fundamentar as transformações que estão em curso em suas práticas - rumo à consolidação das perspectivas da Inclusão Escolar; e, para a consumação de giro linguístico e normativo nos diferentes campos do conhecimento científico e simbólico que definem a deficiência. A Sociologia da Deficiência constitui, portanto, um conjunto de vozes que se devem constituir como parte dos estudos sociológicos da educação contemporânea: vozes por significados e práticas (mais) inclusivas. 


\section{SOCIOLOGY OF DISABILITY: VOICES FOR MEANINGS AND (MORE) INCLUSIVE PRACTICES}

ABSTRACT: In this research, we aim to systematize histories and theories associated to the Sociology of Disability, and, in these systematized elements, identify voices to rethink the meaning of disability and practices of Special Education for disabled people. To do so, a theoretical research was performed, based on bibliographical and documental research, developed from 48 works (papers, essays, books and documents). The texts were primarily compiled from the magazine Disability and Society, from the Asociación Española de Sociología de la Discapacidad and from the database of the Centre of Disability Studies, at the University of Leeds. The compiled works were analyzed using a content analysis technique, called categorical analysis. The first identified category is centered on the main interpretations adopted by the Health Sciences (medical paradigm) to think about disability; the second category is based on the history associated to the establishment of Sociology of Disability - the social movements that (as an answer to interpretations of disability adopted by Health Sciences) (re)signified disability and founded the Disability Studies; the third category focuses on the multiple theoretical faces of Sociology of Disability and on new models of comprehension of disability. From the systematization of these categories' contents, a sociological paradigm was unveiled for disability interpretation. This paradigm presents (as it gathers histories and theories of the Sociology of Disability) more inclusive practices and significances, both in relation to disability and to Special Education.

KEYWORDS: Sociology of disability. Disability studies. Special Education. Disabled people.

\section{REFERÊNCIAS}

ABBERLEY, P. El concepto de opresion y el desarrollo de uma teoria social de la discapacidad. In: BARTON, L. (Org.). Discapacidad y Sociedad. Madrid: Morata, 2008. p.34-50.

Trabalho, Utopía e insuficiência. In: BARTON, L. (Org.). Discapacidad y

Sociedad. Madrid: Morata, 1998. p.81-97.

ASOCIACIÓN ESPAÑOLA DE SOCIOLOGÍA DE LA DISCAPACIDAD

[ASESDIS]. Estatuto. Espanha: ASESDIS, 2002.

BARDIN, L. Análise de conteúdo. 5.ed. Lisboa, PT: Edições70, 2008.

BARNES, C. Un chiste malo: rehabilitar a las personas con discapacidad en una sociedad que discapacita. In: BROGNA, P. (Org.). Visiones y revisiones de la discapacidad. México: FCE, 2009. p.120-143. 
La diferencia producida en una década. Reflexiones sobre la investigación "emancipadora" en discapacidad. In: BARTON, L. (Org.). Superar las barreras de la Discapacidad: 18 años de Disability and Society. Madrid: Morata, 2008. p.381-397.

Disability Studies: What's the Point? Intersticios: Revista Sociológica de Pensamiento Crítico, Madrid, v.1, n.1, p.50-57, 2007.

Vida independiente: Vision Socio-política. In: GARCÍA, A.J.V. (Coord.). El movimiento de Vida Independiente: experiencias internacionales. Madrid: Fundación Luis Vives, 2003. p.61-68. Disponível em: <www.independentliving.org/docs6/alonso2003.pdf>. Acesso em: 7 abr. 2015.

Las teorías de la discapacidad y los orígenes de la opresión de las personas discapacitadas en la sociedad occidental. In: BARTON, L. (Org.). Discapacidad y Sociedad. Madrid: Morata, 1998. p.60-80.

Disabled People in Britain and Discrimination: A Case for AntiDiscrimination Legislation. Canada: Organizations of Disabled People, 1991.

BARNES, C.; OLIVER, M. Discrimination, Disability and Welfare: From Needs to Rights. In: BONYE, I.; BARNES, C.; OLIVER, M. Equal Rights for Disabled People: the case for a new law. Londres: Institute for Public Policy Research, 1991. Disponível em: <http://www.leeds.ac.uk/disabilitystudies/archiveuk/bynoe/equal $\% 20$ rights $\% 20$ for\%20disabled\%20people.pdf $>$. Acesso em: 7 abr. 2015.

BARTON, L. Estudios sobre discapacidad y la búsqueda de la inclusividad. Observaciones. Revista de Educación, Madrid, n.349, p.137-152, may./ago 2009.

BARTON, L. (Org.). Superar las barreras de la Discapacidad. Madrid: Morata, 2008.

(Org.). Discapacidad y Sociedad. Madrid: Morata, 1998.

BROGNA, P. Las representaciones de la Discapacidad: la vigencia del passado en las estruturas sociales presentes. In: BROGNA, P. Visiones e revisions de la discapacidad. México: FCE, 2009. p.157-187.

DÍAZ, E.V. Reflexiones Epistemológicas para uma Sociología de la Discapacidad. Interstícios: Revista Sociológica de Pensamiento Crítico, Madrid, v.3, n.2, p.85-99, 2009.

DISABLED PEOPLE'S INTERNATIONAL [DPI]. The Goals of DPI. 2010. Disponível em: <http://www.dpi.org/AboutUs>. Acesso em: 7 abr. 2015.

FERRANTE, C; FERREIRA, M.A.V. El habitus de la discapacidad: la experiencia corporal de la dominación en un contexto económico periférico. Política y sociedad, Madrid, v.47, n.1, p.85-104, 2010. 
Cuerpo, discapacidad y trayectorias sociales: dos estudios de caso comparados.

Revista de Antropología Experimental, Jaén, v.29, n.8, p.403-428, 2008.

Cuerpo y habitus: el marco estructural de la experiencia de la discapacidad.

2007. Disponível em:

<http://www.um.es/discatif/PROYECTO_DISCATIF/Documentos/Ferrante_Ferreira.pd f>. Acesso em: 22 abr. 2015.

FERREIRA, M.A.V. De la minus-valía a la diversidad funcional: un nuevo marco teórico-metodológico. Política e Sociedad, Madrid, v.47, n.1, p.45-65, 2010.

Una aproximación sociológica a la discapacidad desde el modelo social:

apuntes caracteriológicos. Revista Española de Investigaciones Sociológicas, Madrid, n.124, p.141-174, 2008.

. Sociología de la discapacidad: investigación y compromiso. In: CONGRESO NACIONAL UNIVERSIDAD Y DISCAPACIDAD, 3., 2007, Zaragoza. Anales... Zaragoza, 24-26 oct. 2007.

FERREIRA, M. A. V.; RODRIGUEZ, M. J. C. Sociología de la discapacidad: una propuesta teórica crítica. Nómadas: Revista Crítica de Ciencias Sociales e Jurídicas, Madrid, v.13, n.1, p.243-249, 2006.

FINKELSTEIN, V. The Social Model Of Disability Repossessed. Manchester Coalition of Disabled People, Manchester, p.10-16, fev. 2007. Disponível em: <http://disability-studies.leeds.ac.uk/files/library/finkelstein-soc-mod-repossessed.pdf >. Acesso em: 27 abr. 2015.

. Attitudes and Disabled People: Issues for Discussion. Inglaterra: World Rehabilitation Fund, 1980.

FOUCAULT, M. Vigiar e punir: nascimento da prisão. Tradução de Raquel Ramalhete. 35.ed. Petrópolis: Vozes, 2008.

Os anormais. São Paulo: Martins Fontes, 2001.

GARCIA, A.J.V. Introducion. In: GARCÍA ALONSO, J.V. (Coord.). El movimiento de Vida Independiente: experiencias internacionales. Madrid: Fundación Luis Vives, 2003. p.29-59. Disponível em: <www.independentliving.org/docs6/alonso2003.pdf>. Acesso em: 7 abr. 2015.

GOFFMAN, E. Estigma: notas sobre a manipulação da identidade deteriorada. Rio de Janeiro: Zahar, 1975.

Manicômios, Prisões e Conventos. São Paulo: Perspectiva, 1974.

HANNA, W. J.; ROGOVSKY, B. Mujeres con discapacidad: La suma de dos obstáculos. In: BARTON, L. (Org.). Superar las barreras de a Discapacidad: 18 años de Disability and Society. Madrid: Morata, 2008. p.51-67. 
HUGHES, B.; PATERSON, K. El modelo social de discapacidad y la desaparición del cuerpo: hacia una sociología del impedimento. In: BARTON, L. (Org.). Superar las barreras de la Discapacidad. Madrid: Morata, 2008. p.107-123.

HUNT, P. Settling Accounts With The Parasite People. Disability Challenge, London, n.1, p.37-50, may 1981. Disponível em: <http://disability-

studies.leeds.ac.uk/files/library/UPIAS-Disability-Challenge1.pdf>. Acesso em: 27 abr. 2015 .

(Org.). Stigma: The Experience of Disability. London: Geoffrey, 1966.

LÓPEZ, M.G. Modelos teóricos e investigación en el ámbito de la discapacidad. Hacia la incorporación de la experiencia personal, Córdoba, v.31, n.16, p.215-240, 2006.

MORRIS, J. Lo personal y lo político: una perspectiva feminista sobre la investigación de la discapacidad física. In: BARTON, L. (Org.). Superar las barreras de la Discapacidad. Madrid: Morata, 2008. p.315-326.

OLIVER, M. ¿Están cambiando las relaciones sociales de la producción investigadora? In: BARTON, L. (Org.). Superar las barreras de la Discapacidad: 18 años de Disability and Society. Madrid: Morata, 2008. p.299-314.

- ¿Una sociología de la discapacidad o una sociología discapacitada? In:

BARTON, L. (Org.). Discapacidad y Sociedad. Madrid: Morata, 1998. p.34-59.

. Understanding disability: From theory to practice. New York: St. Martin's Press, 1996.

Capitalism, Disability and Ideology: A Materialist Critique of the Normalization Principle. New York: St. Martin's Press, 1994.

The Politics of Disablement. London: Macmillian, 1990.

PALACIOS, A.; ROMAÑACH, J. El modelo de la diversidad. Interstícios: Revista Sociológica de Pensamiento Crítico, Madrid, v.2, n.2, p.37-47, 2008.

El modelo de la diversidad: la Bioética y los Derechos Humanos como herramientas para alcanzar la plena dignidad en la diversidad funcional. Vedra, Espanha: Ediciones Diversitas, 2006.

PARSONS, T. Social Structure and Dynamic Process: the Case of Modern Medical Practice. In: PARSONS, T. The Social Sistem. 3.ed. Londres: Taylor, 2005. p.31-44.

PETERS, S. ¿Existe una cultura de la discapacidad?: un sincretismo de tres cosmovisiones posibles. In: BARTON, L. (Org.). Superar las barreras de a Discapacidad: 18 años de Disability and Society. Madrid: Morata, 2008. p.142-164.

La política de la identidad de la discapacidad. In: BARTON, L. (Org.).

Discapacidad y Sociedad. Madrid: Moratas, 1998. p.231-251. 
PFEIFFER, D. The philosophical foundations of disability studies. Disability Studies Quarterly, Columbus, v.22, n.2, p.3-23, 2002. Disponível em: <http://www.dsqsds.org/article/view/341/429>. Acesso em: 7 abr. 2015.

RODRÍGUEZ, S.D.; FERREIRA, M.A.V. Diversidad Funcional: sobre lo normal y lo patológico en la condición social de la discapacidad. Cuadernos de Relaciones Laborales, Madrid, v.28, n.1, p.151-172, 2010. Disponível em: <http://revistas.ucm.es/rla/11318635/articulos/CRLA1010120151A.PDF>. Acesso em: 7 abr. 2015.

SHAKESPEARE, T. La autoorganización de las personas con discapacidad: ¿un nuevo movimiento social?. In: BARTON, L. (Org.). Superar las barreras de la

Discapacidad: 18 años de Disability and Society. Madrid: Morata, 2008, p.68-85. 1997.

Defending the Social Model. Disability e Society, Oxon, v.12, n.2, p.293-300,

SHAKESPEARE, T.; WATSON, N. The Social Model of Disability: an outdated ideology? Research in Social Science and Disability, Gateshead, v.2, p.9-28, 2002. Disponível em: <http://www.leeds.ac.uk/disabilitystudies/archiveuk/Shakespeare/social\%20model\%20of\%20disability.pdf $>$. Acesso em: 7 abr. 2015.

TAYLOR, S. et al. (Org.). Disability Studies: Intormation and Resources. 2009. Disponível em: <http://thechp.syr.edu/wpcontent/uploads/2013/10/Disability_Studies_2003_current.pdf >. Acesso em: 7 abr. 2015 .

THOMAS, C. How is disability understood?: an examination of sociological approaches. Disability \& Society, Oxon, v.19, n.6, p.569-583, 2004.

UNION OF THE PHYSICALLY IMPAIRED AGAINST SEGREGATION [UPIAS]. The Union of the Physically Impaired Against Segregation and The Disability Alliance discuss Fundamental Principles of Disability. Londres: UPIAS, 1976.

WORLD HEALTH ORGANIZATION [WHO]. International Classification of Functioning, Disability and Health. Geneva: World Health Organization, 2001. 